\title{
Dabigatran Dual Therapy vs Warfarin Triple Therapy Post-Percutaneous Coronary Intervention in Patients with Atrial Fibrillation With/Without a Proton Pump Inhibitor: A Pre-Specified Analysis of the RE-DUAL PCI Trial
}

\author{
José C. Nicolau ${ }^{1}$ (1) - Deepak L. Bhatt ${ }^{2}$ - Stefan H. Hohnloser ${ }^{3} \cdot$ Takeshi Kimura $^{4}$. Gregory Y. H. Lip ${ }^{5,6}$. Corinna Miede ${ }^{7}$. \\ Matias Nordaby ${ }^{8} \cdot$ Jonas Oldgren $^{9} \cdot$ Philippe Gabriel Steg $^{10,11}$ • Jurriën M. ten Berg ${ }^{12} \cdot$ Lucas C. Godoy $^{1,13}$. \\ Christopher P. Cannon ${ }^{2}$ on behalf of The RE-DUAL PCI Steering Committee and Investigators
}

Published online: 19 June 2020

(c) The Author(s) 2020

\begin{abstract}
Background and Objective In patients with atrial fibrillation following percutaneous coronary intervention, if a proton pump inhibitor is used, could that allow the use of warfarin triple therapy, or is there additional reduction in bleeding while using it with dual therapy?

Methods The RE-DUAL PCI trial randomized 2725 patients with atrial fibrillation post-percutaneous coronary intervention to dabigatran dual therapy (110 or $150 \mathrm{mg}$ twice daily, with clopidogrel or ticagrelor) or warfarin triple therapy (with clopidogrel or ticagrelor, and aspirin for 1-3 months). This prespecified subgroup analysis evaluated risks of a first major bleeding event or clinically relevant non-major bleeding event, all gastrointestinal bleeding, and a composite efficacy endpoint of allcause mortality/thromboembolic event or unplanned revascularization according to baseline use of a proton pump inhibitor. Results Of 2678 analyzed patients, 1641 (61.3\%) were receiving a proton pump inhibitor at baseline. Dabigatran 110 and $150 \mathrm{mg}$ dual therapy reduced the risk of major bleeding events or clinically relevant non-major bleeding events vs warfarin triple therapy regardless of proton pump inhibitor use, with comparable risk of the composite efficacy endpoint (all interaction $p$ values $>0.05$ ). For gastrointestinal bleeding, no interaction was observed between study treatment and proton pump inhibitor use (interaction $p$ values 0.84 and 0.62 for dabigatran 110 and $150 \mathrm{mg}$ dual therapy, respectively, vs warfarin triple therapy). Conclusions Dabigatran 110 and $150 \mathrm{mg}$ dual therapy reduced the risk of major bleeding events or clinically relevant nonmajor bleeding events vs warfarin triple therapy, regardless of proton pump inhibitor use at baseline, in patients with atrial fibrillation who underwent percutaneous coronary intervention. Risk of the composite efficacy endpoint appeared to be similar for dabigatran dual therapy vs warfarin triple therapy in patients receiving/not receiving a proton pump inhibitor.

ClinicalTrials.gov unique identifier NCT02164864.
\end{abstract}

\section{Introduction}

Proton pump inhibitors (PPIs) are recommended for the prevention of gastrointestinal (GI) bleeding in cardiovascular

Digital features To view digital features for this article, go to https://doi.org/10.6084/m9.figshare.12251477.

Electronic supplementary material The online version of this article (https://doi.org/10.1007/s40265-020-01323-x) contains supplementary material, which is available to authorized users.

José C. Nicolau

jose.nicolau@incor.usp.br

Extended author information available on the last page of the article patients receiving antithrombotic therapy, especially in those with a higher risk of bleeding $[1,2]$. Proton pump inhibitors reduce bleeding events when administered with aspirin [3] or clopidogrel [4]; however, results are conflicting on the interactions of PPIs with antiplatelet drugs and effects on clinical outcomes [5-7], and there are concerns regarding their cardiovascular safety $[8,9]$. The Clopidogrel and the Optimization of Gastrointestinal Events Trial is the only randomized study testing PPIs in patients taking dual antiplatelet therapy (DAPT) with aspirin and clopidogrel and, despite the limitations of the trial (mainly the low number of events and the final reduced sample size), it suggested that use of omeprazole reduces upper GI bleeding in patients receiving DAPT, without an increase in cardiovascular events [10]. 


\section{Key Points}

The RE-DUAL PCI trial investigated patients with atrial fibrillation post-percutaneous coronary intervention.

Dabigatran dual therapy (DDT) reduced bleeding risk vs warfarin triple therapy (WTT)

This subanalysis investigated patients categorized according to proton pump inhibitor use at baseline

Clinical efficacy endpoints were similar in DDT and WTT groups regardless of proton pump inhibitor use. DDT reduced bleeding risk vs WTT regardless of proton pump inhibitor use

Proton pump inhibitors are also expected to reduce the risk of GI bleeding in patients receiving oral anticoagulation therapy, as suggested by observations in subjects treated with warfarin [11] and direct oral anticoagulants (DOACs) [12], including dabigatran [13]. Specifically, in patients with atrial fibrillation (AF) who underwent recent percutaneous coronary intervention (PCI), clinical practice guidelines recommend (except in patients with a very high risk of bleeding and a low risk of ischemic events) triple antithrombotic therapy with DAPT and an anticoagulant agent. This regimen further increases the bleeding risk in this population $[1,2,14]$. Regarding dabigatran, a $15 \%$ decrease in drug exposure by PPI use was found in the Randomized Evaluation of Long-term Anticoagulant Therapy (RE-LY) trial, without an observable impact on efficacy or safety [15].

The randomized RE-DUAL PCI trial compared dual antithrombotic therapy with dabigatran 110 or $150 \mathrm{mg}$ twice daily (plus either clopidogrel or ticagrelor) with triple antithrombotic therapy with warfarin (plus aspirin and clopidogrel or ticagrelor) in patients with AF who underwent PCI [16]. Dabigatran dual therapy reduced the risk of the primary endpoint of International Society on Thrombosis and Haemostasis (ISTH)—defined major bleeding events (MBEs) or clinically relevant non-major bleeding events (CRNMBEs) vs warfarin triple therapy, and was non-inferior for the main clinical efficacy endpoint [16].

The effect of PPI administration in patients receiving both anticoagulant and antiplatelet therapies was not studied in the original publication, and it is not known whether the benefits in bleeding reduction with dabigatran dual therapy are valid both for patients taking and those not taking a PPI. This prespecified analysis of the RE-DUAL PCI trial was conducted to investigate whether PPI utilization at baseline could influence the main safety and efficacy results observed in the trial.

\section{Methods}

\subsection{Patient Population}

The RE-DUAL PCI trial (NCT02164864) was a prospective multicenter international study that randomized 2725 patients to dabigatran dual therapy (plus clopidogrel or ticagrelor) or warfarin triple therapy (plus clopidogrel or ticagrelor and aspirin), including 1641 patients using PPIs at randomization. RE-DUAL PCI enrollment criteria, as well as the main trial results, have been published [16, 17]. Patients with paroxysmal, persistent, or permanent non-valvular AF who had undergone successful PCI in the previous 120 hours were eligible. Main exclusion criteria were the presence of creatinine clearance $<30 \mathrm{~mL} / \mathrm{min}$, bioprosthetic or mechanical heart valve, or other major coexisting conditions, such as cardiogenic shock during the current hospitalization, GI bleeding in the previous month, acute liver disease, or contraindication to oral anticoagulation and/or antiplatelet therapy with clopidogrel, ticagrelor, or aspirin.

Patients from the USA and those aged $<80$ years (aged $<70$ years in Japan) were randomized to dabigatran 110 or $150 \mathrm{mg}$ or warfarin in a 1:1:1 ratio. Patients outside the USA aged $\geq 80$ years (aged $\geq 70$ years in Japan) were randomized to the $110-\mathrm{mg}$ dose of dabigatran vs warfarin in 1:1 ratio. In the warfarin triple-therapy group, aspirin was to be discontinued after 1 month in patients with a bare-metal stent implanted and after 3 months when a drug-eluting stent was implanted. All patients were to receive clopidogrel $(75 \mathrm{mg}$ once daily) or ticagrelor (90 mg twice daily) for $\geq 12$ months after randomization; the choice of agent was left to the discretion of the investigator. Regarding PPI use, the study protocol stated: "The concomitant administration of PPI is strongly recommended in patients without clinical contraindications for those agents, to reduce the risk of GI bleeding. In patients receiving clopidogrel, PPIs that do not interact with the CYP2C19 (lansoprazole, pantoprazole, etc.) should be used".

For the present study, 2678 patients with complete data on PPI use at baseline (98.3\% of the total randomized population) were analyzed. The main objective of this prespecified subgroup analysis was to evaluate if PPI use influenced the treatment effect of dabigatran dual vs warfarin triple therapy on ISTH MBEs or CRNMBEs. Secondary objectives included whether PPI use influenced the treatment effect of dabigatran dual vs warfarin triple therapy on ISTH MBEs, all GI bleeding, and all-cause mortality/thromboembolic event (DTE) or unplanned revascularization (clinical efficacy composite endpoint). A further objective was a treatment-independent evaluation (whole population), analyzing the influence of PPI utilization at baseline on bleeding and clinical efficacy composite endpoints. 


\subsection{Statistical Analyses}

Baseline characteristics of the population are summarized for patients taking/not taking PPIs. Continuous variables are depicted as means (standard deviations) and categorical variables as counts (percentages).

Cox proportional hazard regression models stratified by age (non-elderly or elderly; $<70$ or $\geq 70$ years in Japan and $<80$ or $\geq 80$ years elsewhere) were utilized to compare dabigatran $110 \mathrm{mg}$ dual therapy with warfarin triple therapy within PPI subgroup categories. Unstratified Cox proportional hazard regression models were utilized to compare dabigatran $150 \mathrm{mg}$ dual therapy with warfarin triple therapy excluding elderly patients outside the USA. Exploratory treatment by PPI subgroup interaction $p$ values were derived from Cox proportional hazard regression models stratified by age for dabigatran $110 \mathrm{mg}$ dual therapy vs warfarin triple therapy and unstratified for dabigatran $150 \mathrm{mg}$ dual therapy vs warfarin triple therapy. The same subgroup analysis was performed restricting the patient population to patients receiving clopidogrel at baseline.

Additionally, a multivariable adjusted Cox proportional hazard regression model was performed to compare treatment groups including treatment and PPI use at baseline as a factor as well as the interaction between treatment and PPI use at baseline. For bleeding endpoints, this Cox model was adjusted for known bleeding risk factors: continuous variables, age, and creatinine clearance; and categorical variables, prior MBE or bleeding predisposition, prior GI bleeding or ulcerative GI disease or gastritis, diabetes mellitus, aspirin at baseline, modified HAS-BLED score at baseline $(<3$ or $\geq 3$ ), previous stroke, indication for PCI (acute coronary syndrome or elective PCI), and ticagrelor use at baseline. For the clinical efficacy composite endpoint, this Cox model was adjusted for known risk factors of DTE: continuous variables, age, and creatinine clearance; and categorical variables, prior myocardial infarction, diabetes, previous stroke, multi-vessel disease, indication for PCI, and ticagrelor use at baseline. The Cox model to compare dabigatran $110 \mathrm{mg}$ dual therapy with warfarin triple therapy was stratified by age, whereas an unstratified Cox model was used to compare dabigatran $150 \mathrm{mg}$ dual therapy with warfarin triple therapy.

To analyze the role of PPI utilization at baseline on clinical outcomes in the global population, a univariable, treatment-independent, Cox proportional hazard regression analysis stratified by age was developed (non-elderly vs elderly) including PPI use at baseline as the only factor in the model. A multivariable Cox proportional hazard regression analysis comparing risk of clinical outcomes between patients with and without PPI use at baseline was also performed. For bleeding endpoints, the Cox model was adjusted for the above-mentioned bleeding risk factors. For the clinical efficacy composite endpoint, the Cox model was adjusted for risk factors of all-cause mortality and thromboembolic events.

All statistical analyses were two-sided and a $p$ value $<0.05$ was considered statistically significant. All $p$ values are to be considered as exploratory. SAS 9.4 (SAS Institute Inc., Cary, NC, USA) was used for all statistical analyses.

\section{Results}

Of 2678 analyzed patients, 1641 (61.3\%) used PPIs at baseline (Fig. 1 of the Electronic Supplementary Material [ESM]). Table 1 shows the characteristics of the population. Patients taking PPIs comprised a higher-risk population, being more frequently female and with a prior history of myocardial infarction, PCI, diabetes, hypertension, and GI bleeding. Mean $\mathrm{CHA}_{2} \mathrm{DS}_{2-} \mathrm{VASc}$ and modified HASBLED scores were similar between groups. Table 2 of the ESM shows the baseline characteristics of the population by PPI use and further split by treatment (i.e., eight groups). Regarding age, it is important to remember that patients outside the USA aged older than 79 years (aged older than 69 years in Japan) were precluded to receive dabigatran $150 \mathrm{mg}$ dual therapy. As can be seen in Fig. 2 of the ESM, the percentage of patients taking PPIs was stable during the followup. For the 1641 patients taking PPIs at baseline, information on PPI use at the end-of-treatment visit was missing for 75 patients. Of the remaining 1566 patients, 1306 (83.4\%) took PPIs at the end-of-treatment visit or since the last visit before the end of treatment. Of the 984 patients not using PPIs at baseline with available information at the end-oftreatment visit, $23.1 \%$ were taking PPIs at the end-of-treatment visit or since the last visit before the end of treatment (for 53 patients, information was missing at this visit).

\subsection{Influence of Proton Pump Inhibitor Utilization at Baseline Regarding the Comparison of Dabigatran Dual vs Warfarin Triple Therapy}

Dabigatran 110 and $150 \mathrm{mg}$ dual therapy consistently reduced the risk of ISTH MBEs/CRNMBEs vs warfarin triple therapy regardless of PPI use, with a comparable risk for the composite efficacy endpoint.

Figure 1 shows the population receiving dabigatran $110 \mathrm{mg}$ dual therapy compared with warfarin triple therapy. Dabigatran $110 \mathrm{mg}$ dual therapy considerably reduced the risk of bleeding events (ISTH MBE/CRNMBE, ISTH MBE alone) vs warfarin triple therapy, with a comparable risk of the clinical composite efficacy endpoint in both treatment groups, independent of PPI use at baseline ( $p$ values for interaction $>0.05$ ).

Figure 2 analyzes the same parameters for the population receiving dabigatran $150 \mathrm{mg}$ dual therapy. The group 
dabigatran dual therapy considerably reduced the risk of ISTH MBEs/CRNMBEs vs warfarin triple therapy with a comparable risk of the clinical composite efficacy endpoint, independent of PPI utilization ( $p$ values for interaction $>0.05$ ).

Figure 3 shows the additional endpoint of any GI bleeding. No statistically significant interaction between treatment and PPI use at baseline was observed comparing dabigatran dual therapy with warfarin triple therapy (interaction $p$ values 0.84 and 0.62 for dabigatran 110 and $150 \mathrm{mg}$ dual therapy, respectively).

The results from multivariable-adjusted Cox models are similar to those in Figs. 1, 2 and 3 ( $p$ values for interaction $>0.05$, Table 2). Importantly, the multivariable-adjusted hazard ratios of GI bleeding for dabigatran $110 \mathrm{mg}$ dual therapy vs warfarin triple therapy were 0.82 (95\% confidence interval $[\mathrm{CI}]$ 0.42-1.59) for patients using PPIs and

Table 1 Characteristics of the RE-DUAL PCI population: proton pump inhibitor (PPI) vs no PPI at baseline

\begin{tabular}{|c|c|c|}
\hline & $\begin{array}{l}\text { PPI at baseline, yes } \\
\text { Total }(n=1641)\end{array}$ & $\begin{array}{l}\text { PPI at baseline, no } \\
\text { Total }(n=1037)\end{array}$ \\
\hline \multicolumn{3}{|l|}{ Demographic data } \\
\hline Age, mean (SD), years & $71.0(8.7)$ & $70.4(8.6)$ \\
\hline Male sex, $n(\%)$ & $1220(74.3)$ & $813(78.4)$ \\
\hline $\mathrm{CrCl}, \mathrm{mL} / \mathrm{min}$, mean $(\mathrm{SD})^{\mathrm{a}}$ & $76.5(29.5)$ & $80.5(29.3)$ \\
\hline \multicolumn{3}{|l|}{ Medical and surgical history } \\
\hline Prior MI, $n(\%)$ & $458(27.9)$ & $229(22.1)$ \\
\hline Prior CAD, $n(\%)$ & $1096(66.8)$ & $701(67.6)$ \\
\hline Prior PCI, $n(\%)$ & $585(35.6)$ & $311(30)$ \\
\hline LV ejection fraction, ${ }^{\mathrm{b}}$ mean (SD), \% & $50.6(12.3)$ & $51.7(12.8)$ \\
\hline Heart failure, $n(\%)$ & $570(34.7)$ & $350(33.8)$ \\
\hline Diabetes mellitus, $n(\%)$ & $615(37.5)$ & $358(34.5)$ \\
\hline Hypertension, $n(\%)$ & $912(55.6)$ & $479(46.2)$ \\
\hline Currently smoking, ${ }^{\mathrm{c}} n(\%)$ & $214(13.0)$ & $120(11.6)$ \\
\hline Previous GI bleeding, ulcerative GI disease, or gastritis, $n(\%)$ & $135(8.2)$ & $44(4.2)$ \\
\hline Prior major bleeding or predisposition to bleeding, $n(\%)$ & $18(1.1)$ & $13(1.3)$ \\
\hline $\mathrm{CHA}_{2} \mathrm{DS}_{2}$-VASc score, mean (SD) & $3.7(1.6)$ & $3.5(1.6)$ \\
\hline Modified HAS-BLED score, mean (SD) & $2.7(0.7)$ & $2.7(0.7)$ \\
\hline \multicolumn{3}{|l|}{ In-hospital approach } \\
\hline Indication for PCI: ACS, $n(\%)$ & $875(53.3)$ & $478(46.1)$ \\
\hline Radial access, ${ }^{\mathrm{d}} n(\%)$ & $1054(64.2)$ & $649(62.6)$ \\
\hline Only one coronary stent, ${ }^{\mathrm{e}} n(\%)$ & $1326(80.8)$ & $816(78.7)$ \\
\hline Use of DES only, ${ }^{\mathrm{f}} n(\%)$ & $1367(83.3)$ & $843(81.3)$ \\
\hline UFH use in index procedure, $n(\%)$ & $1396(85.1)$ & $859(82.8)$ \\
\hline \multicolumn{3}{|l|}{ Baseline medications } \\
\hline Clopidogrel, $n(\%)$ & $1451(88.4)$ & $880(84.9)$ \\
\hline Statins & $1455(88.7)$ & $879(84.8)$ \\
\hline Oral hypoglycemic drugs & $430(26.2)$ & $255(24.6)$ \\
\hline ACE inhibitors & $912(55.6)$ & $497(47.9)$ \\
\hline Angiotensin receptor blockers & $535(32.6)$ & $334(32.2)$ \\
\hline$\beta$-blockers & $1387(84.5)$ & $844(81.4)$ \\
\hline
\end{tabular}

Note, 47 patients with missing information on PPI use at baseline are excluded

$A C E$ angiotensin converting enzyme, $A C S$ acute coronary syndrome, $C A D$ coronary artery disease, $C r C l$ creatinine clearance, $D E S$ drug-eluting stent, $G I$ gastrointestinal, $L V$ left ventricular, $M I$ myocardial infarction, $P C I$ percutaneous coronary intervention, $S D$ standard deviation, $U F H$ unfractionated heparin

${ }^{\text {a }}$ Missing in 221 patients

${ }^{\mathrm{b}}$ Missing in 183 patients

${ }^{\mathrm{c}}$ Missing in one patient

${ }^{\mathrm{d}}$ Missing in 20 patients

${ }^{\mathrm{e}}$ Missing in 50 patients

${ }^{\mathrm{f}}$ Missing in seven patients 
0.70 (95\% CI 0.29-1.71) for those not using PPIs ( $p$-interaction $=0.78)$; the hazard ratios for the comparison between dabigatran $150 \mathrm{mg}$ dual therapy and warfarin triple therapy were, respectively, 0.83 (95\% CI 0.36-1.91) and 0.71 (95\% CI 0.25-2.06) [ $p$-interaction $=0.81]$. Table 1 of the ESM shows the same analyses as in Figs. 1, 2 and 3 but restricted to patients taking clopidogrel ( $87 \%$ of the population). Similar to that global population's results, the $p$ values obtained for interaction were all $>0.05$. Specifically for GI bleeding, the $p$ values for interaction were 0.61 for the comparison between dabigatran $110 \mathrm{mg}$ dual therapy and warfarin triple therapy and 0.35 for the comparison between dabigatran 150-mg dual therapy and warfarin triple therapy.

\subsection{Influence of Proton Pump Inhibitor Utilization at Baseline on the Bleeding and Clinical Efficacy Composite Endpoint in the Whole Population (Study Treatment-Independent Analysis)}

In an additional analysis, we evaluated event rates for the whole population among patients treated or not treated with PPIs, by means of both univariable and multivariable models. The univariable analyses showed hazard ratios [HRs; 95\% CIs of 1.00 (0.84-1.18), 1.12 (0.82-1.52), 1.23 (0.81-1.89), and 1.20 (0.96-1.48)] for ISTH MBE/ CRNMBE, ISTH MBE alone, GI bleeding, and DTE/ unplanned revascularization, respectively. The multivariable-adjusted analyses showed similar results, with HRs $(95 \%$ CIs) of 0.93 (0.78-1.12) for ISTH MBEs/CRNMBEs, 1.13 (0.72-1.77) for GI bleeding, and $1.16(0.92-1.45)$ for the clinical efficacy composite endpoint (Fig. 4).

\section{Discussion}

In this prespecified analysis of the RE-DUAL PCI trial [16] in patients with AF undergoing PCI, the benefit of dual antithrombotic therapy with dabigatran 110 or $150 \mathrm{mg}$ twice daily in reducing the risk of MBEs or CRNMBEs, when compared with triple therapy with warfarin, did not appear to be influenced by use of a PPI at baseline. As such, dual therapy with both dosages of dabigatran did not seem to differ from warfarin triple therapy in terms of the risk of experiencing DTE or unplanned revascularization, also irrespective of PPI administration.

A meta-analysis comparing dual with triple antithrombotic therapy in 5317 patients from four randomized controlled trials (including RE-DUAL PCI) showed that dual therapy led to lower risks of major or minor bleeding (HR, 0.53 ; 95\% CI 0.36-0.85) per Thrombolysis in Myocardial Infarction (TIMI) Study Group criterion, with comparable risk of major adverse cardiovascular events (HR 0.85; 95\%
Fig. 1 Safety and efficacy outcomes in patients receiving/not receiving proton pump inhibitors (PPIs) at baseline: dabigatran 110-mg dual therapy vs warfarin triple therapy. Hazard ratios (HRs) and 95\% confidence interval (CIs) from the Cox proportional hazard model; stratified by age (elderly vs nonelderly). CRNMBE clinically relevant non-major bleeding event, DTE death/ thromboembolic event, ISTH International Society on Thrombosis and Haemostasis, $M B E$ major bleeding event

\begin{tabular}{ccccc}
\multicolumn{2}{c}{$n / N(\%)$} & & HR, $95 \% \mathrm{Cl}$ \\
Dabigatran & Warfarin & & Dabigatran $110 \mathrm{mg}$ \\
Outcome & $110 \mathrm{mg}$ & Triple & HR & Dual Therapy Versus \\
Wual Therapy & Therapy & $(95 \% \mathrm{Cl})$ & Warfarin Triple Therapy
\end{tabular}

\begin{tabular}{llll}
\multicolumn{4}{l}{ ISTH MBE or CRNMBE } \\
PPI & $98 / 606(16.2)$ & $153 / 580(26.4)$ & $0.56(0.44-0.73)$ \\
No PPI & $52 / 366(14.2)$ & $106 / 368(28.8)$ & $0.44(0.32-0.62)$
\end{tabular}

Interaction $p$ value: 0.2617

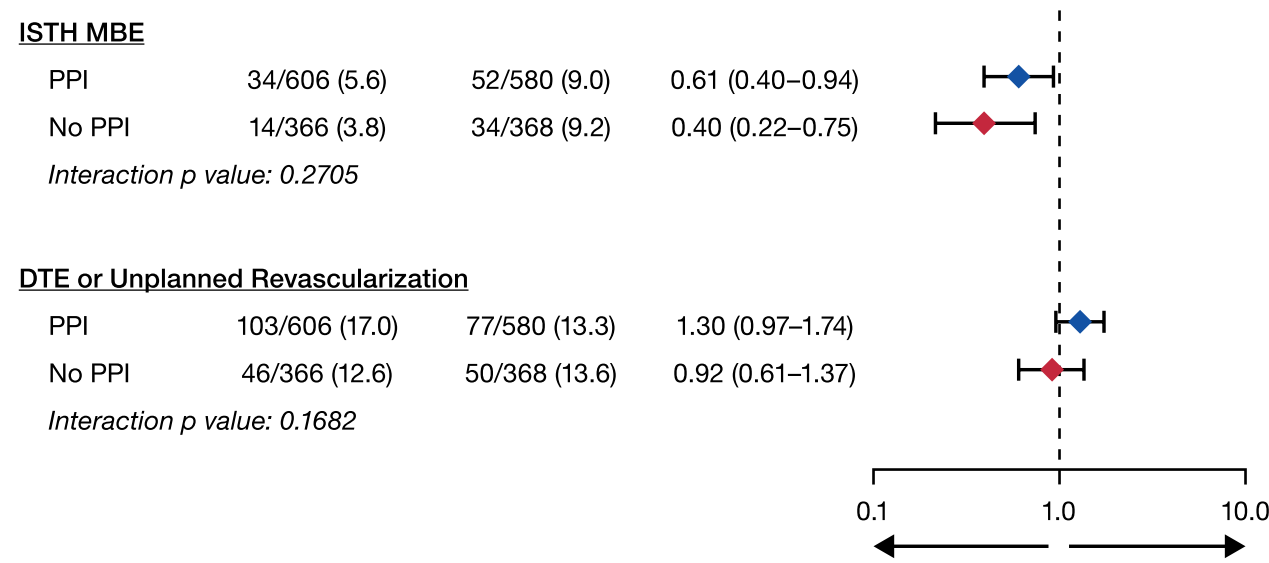

Favors Dabigatran Favors Warfarin $110 \mathrm{mg}$ Dual Therapy Triple Therapy 
Table 2 Safety and efficacy outcomes in patients receiving/not receiving proton pump inhibitors (PPIs) at baseline. Dabigatran dual therapy vs warfarin triple therapy: multivariable-adjusted analyses

\begin{tabular}{|c|c|c|c|c|c|c|c|}
\hline & $\begin{array}{l}\text { PPI at } \\
\text { baseline }\end{array}$ & $\begin{array}{l}\text { Dabigatran } \\
110 \mathrm{mg} \text { dual } \\
\text { therapy } n / N(\%)\end{array}$ & $\begin{array}{l}\text { Warfarin triple } \\
\text { therapy } \\
n / N(\%)\end{array}$ & $\operatorname{HR}(95 \%)$ & $\begin{array}{l}\text { Dabigatran } \\
150 \mathrm{mg} \text { dual } \\
\text { therapy } n / N(\%)\end{array}$ & $\begin{array}{l}\text { Warfarin triple } \\
\text { therapy } \\
n / N(\%)^{\mathrm{b}}\end{array}$ & $\operatorname{HR}(95 \%)$ \\
\hline \multirow{3}{*}{$\begin{array}{l}\text { Adjudicated } \\
\text { ISTH MBE/ } \\
\text { CRNMB }\end{array}$} & PPI & $89 / 549(16.2)$ & $137 / 527(26.0)$ & $0.64(0.48-0.85)$ & $82 / 417$ (19.7) & $106 / 405(26.2)$ & $0.75(0.54-1.03)$ \\
\hline & No PPI & 48/323 (14.9) & $100 / 338(29.6)$ & $0.52(0.36-0.74)$ & $56 / 273(20.5)$ & $73 / 267(27.3)$ & $0.78(0.54-1.13)$ \\
\hline & $\begin{array}{l}\text { Interaction } p \\
\text { value }\end{array}$ & & & 0.3476 & & & 0.8640 \\
\hline \multirow{3}{*}{$\begin{array}{l}\text { Adjudicated } \\
\text { ISTH MBE }\end{array}$} & PPI & $31 / 549(5.6)$ & $45 / 527(8.5)$ & $0.73(0.44-1.20)$ & $22 / 417(5.3)$ & $32 / 405$ (7.9) & $0.65(0.35-1.21)$ \\
\hline & No PPI & $12 / 323(3.7)$ & $32 / 338(9.5)$ & $0.44(0.22-0.88)$ & $14 / 273(5.1)$ & 23/267 (8.6) & $0.63(0.31-1.28)$ \\
\hline & $\begin{array}{l}\text { Interaction } p \\
\text { value }\end{array}$ & & & 0.2204 & & & 0.9228 \\
\hline \multirow{3}{*}{$\begin{array}{l}\text { Adjudicated GI } \\
\text { bleeding }\end{array}$} & PPI & $17 / 549(3.1)$ & $24 / 527(4.6)$ & $0.82(0.42-1.59)$ & $15 / 417(3.6)$ & $15 / 405(3.7)$ & $0.83(0.36-1.91)$ \\
\hline & No PPI & $8 / 323(2.5)$ & $15 / 338(4.4)$ & $0.70(0.29-1.71)$ & $7 / 273(2.6)$ & $9 / 267(3.4)$ & $0.71(0.25-2.06)$ \\
\hline & $\begin{array}{l}\text { Interaction } p \\
\text { value }\end{array}$ & & & 0.7792 & & & 0.8067 \\
\hline \multirow{3}{*}{$\begin{array}{l}\text { Adjudicated } \\
\text { DTE/ } \\
\text { unplanned } \\
\text { revasculariza- } \\
\text { tion }\end{array}$} & PPI & $93 / 544(17.1)$ & $71 / 520(13.7)$ & $1.25(0.92-1.71)$ & $54 / 413(13.1)$ & $56 / 398(14.1)$ & $0.96(0.66-1.40)$ \\
\hline & No PPI & $43 / 327(13.1)$ & 45/332 (13.6) & $0.95(0.62-1.44)$ & $27 / 269(10.0)$ & $30 / 265(11.3)$ & $0.92(0.54-1.55)$ \\
\hline & $\begin{array}{l}\text { Interaction } p \\
\text { value }\end{array}$ & & & 0.2877 & & & 0.8954 \\
\hline
\end{tabular}

$C R N M B E$ clinically relevant non-major bleeding event, DTE death/thromboembolic event, $G I$ gastrointestinal, $H R$ hazard ratio, ISTH International Society on Thrombosis and Haemostasis, $M B E$ major bleeding event

${ }^{a}$ See Sect. 2.2 for details

${ }^{\mathrm{b}}$ For the comparison with dabigatran $150 \mathrm{mg}$ dual therapy, elderly patients outside the USA are excluded

CI 0.48-1.29) [18]. More recently, a network meta-analysis of 10,026 patients from four trials (including RE-DUAL PCI) reported odds ratios for TIMI major bleeding of 0.49 (95\% CI 0.30-0.82) for DOAC plus a P2Y12 inhibitor, 0.58 (95\% CI 0.31-1.08) for a vitamin $\mathrm{K}$ antagonist plus a P2Y12 inhibitor, and 0.70 (95\% CI 0.38-1.23) for DOAC plus DAPT, compared to a vitamin $\mathrm{K}$ antagonist plus DAPT as reference [19]. There was no significant difference in the risk of major adverse cardiovascular events [19].

To our knowledge, ours is the first study to investigate PPI use in patients receiving triple vs dual antithrombotic therapy with DOACs. In patients taking DAPT without anticoagulants, previous reports found that the use of PPIs significantly reduces the risk of GI bleeding [10, 20-22]. Similarly, a retrospective cohort analysis with patients using warfarin and aspirin suggested that PPI use was associated with a lower risk of upper GI bleeding hospitalization (adjusted HR 0.51; 95\% CI 0.27-0.97), with a tendency of benefit for patients taking warfarin and a P2Y12 inhibitor (HR 0.54; 95\% CI 0.29-1.01) [11]. In a large Medicare population taking an anticoagulant agent (very few with concomitant antiplatelet therapy), PPI use was associated with lower rates of hospitalization for upper GI bleeding when coadministered with both warfarin and DOACs, including dabigatran (incidence rate ratio for dabigatran: $0.49 ; 95 \%$ CI 0.41-0.59) [12].
The COMPASS trial analyzed $>17,000$ patients randomized to pantoprazole $40 \mathrm{mg}$ daily or placebo on top of rivaroxaban and aspirin or monotherapy with each drug. In a 3-year follow-up, similar rates of the composite endpoint of cardiovascular deaths, myocardial infarction, or stroke were found for pantoprazole or the placebo groups [23]. The drug was generally well tolerated, with the exception of a significant 33\% increase in the incidence of enteric infection. There were no significant differences between pantoprazole or placebo regarding upper GI events globally, or in other analyzed bleeding parameters, with the exception of "overt bleeding of GI origin confirmed by endoscopy of radiography" (annual rate of $0.060 \%$ for pantoprazole vs $0.12 \%$ for placebo, $p=0.03$ ) [24].

In the RE-LY trial, dabigatran $150 \mathrm{mg}$ twice daily was associated with higher rates of GI bleeding compared with warfarin, which was not observed with dabigatran $110 \mathrm{mg}$ [25]. Dabigatran is administered as a prodrug (dabigatran etexilate) and only $3-7 \%$ is absorbed into the systemic circulation [26, 27]. The remaining non-absorbed prodrug may also undergo intraluminal activation by gut esterases, which may lead to bleeding owing to a topic effect on the intestinal mucosa $[28,29]$. Conversely, bleeding events caused by antiplatelet drugs and warfarin are observed mainly in the upper GI tract [10,30]. Given these differences in anatomic sites of bleeding, PPIs would be expected to have a more significant 
Fig. 2 Safety and efficacy outcomes in patients receiving/not receiving proton pump inhibitors (PPIs) at baseline: dabigatran $150 \mathrm{mg}$ dual therapy vs warfarin triple therapy. For the comparison with $150 \mathrm{mg}$ dabigatran dual therapy, elderly patients outside the USA are excluded. Hazard ratios (HRs) and $95 \%$ confidence intervals (CIs) from the unstratified Cox proportional hazard model. $C R N M B E$ clinically relevant non-major bleeding event, DTE death/thromboembolic event, ISTH International Society on Thrombosis and Haemostasis, $M B E$ major bleeding event

\begin{tabular}{ccccc} 
& \multicolumn{2}{c}{$n / N(\%)$} & & HR, $95 \% \mathrm{Cl}$ \\
Dabigatran & Warfarin & & Dabigatran $150 \mathrm{mg}$ \\
Dutcome & $150 \mathrm{mg}$ & Triple & HR & Dual Therapy Versus \\
Wual Therapy & Therapy & $(95 \% \mathrm{Cl})$ & Warfarin Triple Therapy
\end{tabular}

ISTH MBE or CRNMBE

$\begin{array}{llll}\text { PPI } & 94 / 455(20.7) & 116 / 445(26.1) & 0.73(0.56-0.96) \\ \text { No PPI } & 59 / 303(19.5) & 77 / 292(26.4) & 0.66(0.47-0.92)\end{array}$

Interaction $p$ value: 0.6665

\section{ISTH MBE}

$\begin{array}{llll}\text { PPI } & 27 / 455(5.9) & 36 / 445(8.1) & 0.71(0.43-1.17) \\ \text { No PPI } & 15 / 303(5.0) & 25 / 292(8.6) & 0.54(0.28-1.02)\end{array}$

Interaction $p$ value: 0.5051

DTE or Unplanned Revascularization
$\begin{array}{llll}\text { PPI } & 58 / 455(12.7) & 61 / 445(13.7) & 0.91(0.64-1.31) \\ \text { No PPI } & 31 / 303(10.2) & 33 / 292(11.3) & 0.88(0.54-1.43)\end{array}$

Interaction $p$ value: 0.8922

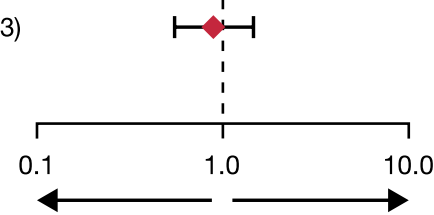

Favors Dabigatran Favors Warfarin 150 mg Dual Therapy Triple Therapy
Fig. 3 Gastrointestinal bleeding in patients receiving/ not receiving proton pump inhibitors (PPIs) at baseline: dabigatran $110 \mathrm{mg}$ and $150 \mathrm{mg}$ dual therapy vs warfarin triple therapy. For the comparison with $150 \mathrm{mg}$ dabigatran dual therapy, elderly patients outside the USA are excluded. Statistics as in Figs. 1 and 2, respectively. $C I$ confidence interval, $H R$ hazard ratio

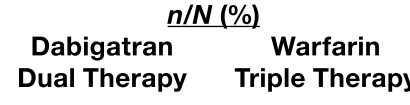

\section{Dabigatran $110 \mathrm{mg}$ Dual Therapy

PPI $\quad$ 19/606 (3.1) \\ No PPI \\ $9 / 366(2.5)$}

Interaction $p$ value: 0.8448

Dabigatran $150 \mathrm{mg}$ Dual Therapy

$\begin{array}{ll}\text { PPI } & 18 / 455(4.0) \\ \text { No PPI } & 8 / 303(2.6)\end{array}$

Interaction $p$ value: 0.6161

$27 / 580(4.7)$

$15 / 368(4.1)$ $\begin{array}{ll}16 / 445(3.6) & 1.08(0.55-2.12) \\ 9 / 292(3.1) & 0.81(0.31-2.09)\end{array}$

$0.66(0.37-1.19)$

$0.61(0.27-1.39)$

HR
$(95 \%$ cI)

Dabigatran Dual Therapy Versus Warfarin Triple Therapy

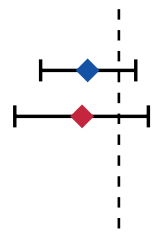

1)

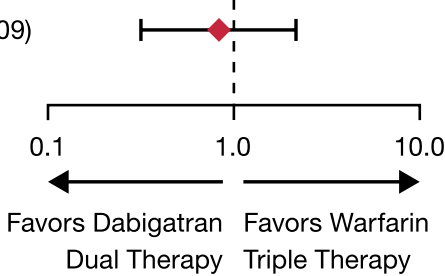

role in bleeding prevention for patients treated with warfarin triple therapy than patients receiving dabigatran dual therapy. Nevertheless, contrary to this rationale, our analysis suggests that, even when receiving a PPI, patients in the warfarin triple-therapy group did not show lower GI bleeding rates than patients prescribed dabigatran dual therapy, with a numerically lower risk for patients utilizing PPIs with dabigatran $110 \mathrm{mg}$ dual therapy, in comparison with warfarin triple therapy plus PPIs (HR of 0.66).

Regarding clinical efficacy (DTE/unplanned revascularization), PPIs may reduce absorption of dabigatran [31, 32], and concerns have been raised that PPIs could reduce the 
Fig. 4 Safety and efficacy outcomes in patients receiving/not receiving proton pump inhibitors (PPIs) at baseline: study treatment-independent multivariable analyses. Hazard ratios (HRs) and confidence intervals (CIs) from a stratified Cox proportional hazard regression analysis. See Sect. 2.2 for details. CRNMBE clinically relevant non-major bleeding event, DTE death/thromboembolic event, ISTH International Society on Thrombosis and Haemostasis, $M B E$ major bleeding event

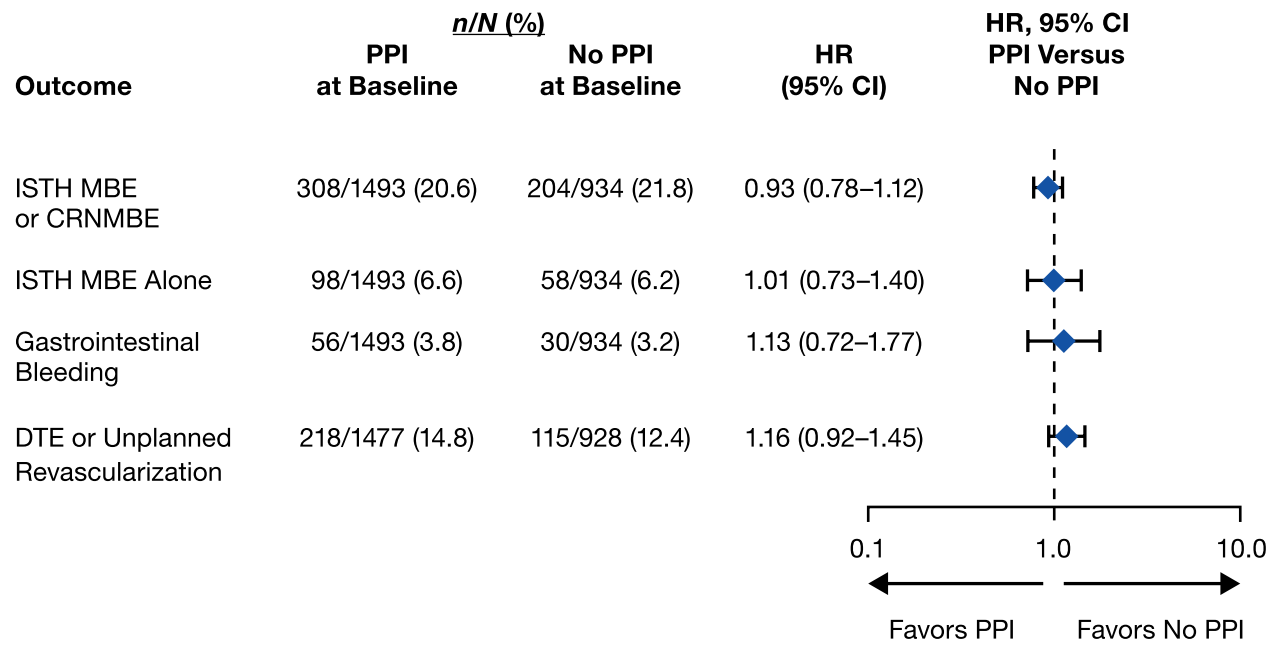

conversion of clopidogrel (the most commonly used P2Y12 inhibitor in RE-DUAL: 87\%) into its active metabolite, [31] despite that in RE-DUAL the benefits of dabigatran $110 \mathrm{mg}$ or $150 \mathrm{mg}$ dual therapy in comparison with warfarin triple therapy in decreasing bleeding risks were consistent across patients treated with clopidogrel or ticagrelor. [33] Proton pump inhibitors could also have a deleterious impact in vascular function per se [9]. All these theoretical effects could eventually lead to a higher incidence of clinical efficacy endpoints in patients treated with a PPI [4, 34, 35].

In the present study, in the dabigatran 110-mg subgroup, a HR of 1.30 was observed for patients taking PPIs vs not taking PPIs, but this trend was not observed in the dabigatran 150-mg subgroup. Thus, no consistent difference between treatment groups was seen. However, despite that no significant interactions were observed in the subgroup of patients taking clopidogrel, a HR of 1.45 was observed in the population on dabigatran $110 \mathrm{mg}$, but this trend was not observed in the dabigatran $150 \mathrm{mg}$ subgroup; this finding could be related to an interaction between PPI and clopidogrel [7], leading to an increase in clinical events in the dabigatran $110 \mathrm{mg}$ subgroup that was blunted with the dabigatran $150 \mathrm{mg}$ doses. However, we urge caution because this one finding is in a subgroup of a subgroup, and the interaction $p$ value is not statistically significant, thus indicating that this cannot be determined from this dataset.

\subsection{Limitations}

Our results should be interpreted in the context of a subgroup analysis of a randomized trial in a setting with very few data available from other sources. The RE-DUAL PCI trial was not powered for any subgroup analysis and no adjustment for multiplicity was performed, thus the reported results should be regarded as exploratory. As in all publications with similar characteristics, there are imbalances in the baseline characteristics of patients taking vs not taking PPIs, and differences in baseline characteristics were previously acknowledged as a possible source of bias when analyzing the effect of PPI therapy on cardiovascular outcomes [4]. Second, we did not have information about the time using a PPI during follow-up, which may limit assessment of the influence of PPI intake on long-term bleeding and cardiovascular outcomes. Third, more granular data regarding PPI brand and dosage were not available, limitations that are shared with similar analyses [12]. Finally, we did not retrieve information about upper vs lower GI bleeding, which could be useful to better understand potential interactions between PPIs, DOAC use, and bleeding.

\section{Conclusions}

Dual therapy with dabigatran at both 110- and 150-mg doses twice daily reduced the risk of bleeding when compared with warfarin triple therapy, regardless of PPI use at baseline in patients with AF undergoing PCI. The risk of clinical efficacy endpoints appeared similar with dabigatran dual therapy vs warfarin triple therapy irrespective of PPI utilization.

Acknowledgements Editorial assistance limited to formatting and figure preparation was provided by Bill Wolvey at Parexel, with funding from Boehringer Ingelheim International $\mathrm{GmbH}$.

Funding The study was funded by Boehringer Ingelheim International $\mathrm{GmbH}$.

\section{Compliance with Ethical Standards}

Conflict of interest José C. Nicolau has received research grants from CSL Behring, Sanofi, AstraZeneca, Bristol-Myers Squibb, Viforpharma, Dalcorpharma, Janssen, and Novartis; has served as a consultant/ advisory board member for Bayer, Merck, Novartis, Sanofi, Servier, Amgen, and AstraZeneca; and a steering committee member for Ei- 
sai, Boehringer Ingelheim, AstraZeneca, Sanofi, CSL Behring, Viforpharma, and Dalcorpharma. Deepak L. Bhatt discloses the following relationships: Advisory Board: Cardax, Cereno Scientific, Elsevier Practice Update Cardiology, Medscape Cardiology, PhaseBio, Regado Biosciences; Board of Directors: Boston VA Research Institute, Society of Cardiovascular Patient Care, TobeSoft; Chair: American Heart Association Quality Oversight Committee; Data Monitoring Committees: Baim Institute for Clinical Research (formerly Harvard Clinical Research Institute, for the PORTICO trial, funded by St. Jude Medical, now Abbott), Cleveland Clinic (including for the ExCEED trial, funded by Edwards), Duke Clinical Research Institute, Mayo Clinic, Mount Sinai School of Medicine (for the ENVISAGE trial, funded by Daiichi Sankyo), Population Health Research Institute; Honoraria: American College of Cardiology (Senior Associate Editor, Clinical Trials and News, ACC.org; Vice-Chair, ACC Accreditation Committee), Baim Institute for Clinical Research (formerly Harvard Clinical Research Institute; RE-DUAL PCI clinical trial steering committee funded by Boehringer Ingelheim; AEGIS-II executive committee funded by CSL Behring), Belvoir Publications (Editor-in-Chief, Harvard Heart Letter), Duke Clinical Research Institute (clinical trial steering committees, including for the PRONOUNCE trial, funded by Ferring Pharmaceuticals), HMP Global (Editor-in-Chief, Journal of Invasive Cardiology), Journal of the American College of Cardiology (Guest Editor; Associate Editor), Medtelligence/ReachMD (CME steering committees), Population Health Research Institute (for the COMPASS operations committee, publications committee, steering committee, and USA national co-leader, funded by Bayer), Slack Publications (Chief Medical Editor, Cardiology Today's Intervention), Society of Cardiovascular Patient Care (Secretary/Treasurer), WebMD (CME steering committees); other: Clinical Cardiology (Deputy Editor), NCDR-ACTION Registry Steering Committee (Chair), VA CART Research and Publications Committee (Chair); Research Funding: Abbott, Afimmune, Amarin, Amgen, AstraZeneca, Bayer, Boehringer Ingelheim, Bristol-Myers Squibb, Chiesi, CSL Behring, Eisai, Ethicon, Ferring Pharmaceuticals, Forest Laboratories, Fractyl, Idorsia, Ironwood, Ischemix, Lilly, Medtronic, PhaseBio, Pfizer, Regeneron, Roche, Sanofi Aventis, Synaptic, The Medicines Company; Royalties: Elsevier (Editor, Cardiovascular Intervention: A Companion to Braunwald's Heart Disease); Site Co-Investigator: Biotronik, Boston Scientific, CSI, St. Jude Medical (now Abbott), Svelte; Trustee: American College of Cardiology; Unfunded Research: FlowCo, Merck, Novo Nordisk, PLx Pharma, Takeda. Stefan H. Hohnloser has received personal fees from Bayer HealthCare, Boehringer Ingelheim, Bristol-Myers Squibb, Daiichi Sankyo, Medtronic, Pfizer, SJM, and ZOLL. Takeshi Kimura has received grants from Boehringer Ingelheim. Gregory Y.H. Lip has served as a consultant for Bayer/Janssen, Bristol-Meyers Squibb/Pfizer, Medtronic, BoehringerIngelheim, Novartis, Verseon, andDaiichiSankyo. $\mathrm{He}$ has been a speaker for Bayer, Bristol-Meyers Squibb/Pfizer, Boehringer Ingelheim, Daiichi Sankyo, and Medtronic. No fees are personally received. Corinna Miede is an employee of Mainanalytics $\mathrm{ma} \mathrm{GmbH}$, contracted by Boehringer Ingelheim International $\mathrm{GmbH}$. Matias Nordaby is an employee of Boehringer Ingelheim International $\mathrm{GmbH}$. Jonas Oldgren has received fees to his institution from AstraZeneca, Bayer HealthCare, Boehringer Ingelheim, Bristol-Myers Squibb, Daiichi Sankyo, Pfizer, Portola, Roche Diagnostics, and Sanofi. Philippe Gabriel Steg has received research grants from Amarin, Bayer, Sanofi, and Servier; speaking or consulting fees from Amarin, Amgen, AstraZeneca, Bayer/Janssen, Boehringer Ingelheim, BristolMyers Squibb, Idorsia, Lilly, Merck, Novartis, Novo Nordisk, Pfizer, Regeneron, Sanofi, and Servier. Jurriën M. ten Berg has received advisory/consulting/speaker fees from Accumetrics, AstraZeneca, Bayer HealthCare, Boehringer Ingelheim, Bristol-Myers Squibb, Daiichi Sankyo, Eli Lily, Ferrer, The Medicines Company, and Pfizer, and has received research grants from AstraZeneca, ZonMw. Lucas C. Godoy has no conflicts of interest that are directly relevant to the content of this article. Christopher P. Cannon has received research grants from
Amgen, Boehringer Ingelheim, Bristol-Myers Squibb, Daiichi Sankyo, Janssen, Merck, and Pfizer and consulting fees from Aegerion, Alnylam, Amarin, Amgen, Applied Therapeutics, Ascendia, BGB, Boehringer Ingelheim, Bristol-Myers Squibb, Corvidia, HLS Therapeutics, Innovent, Janssen, Kowa, Merck, Pfizer, and Sanofi.

Ethics Approval The study was approved by the ethics committee at each participating center and all procedures were performed in accordance with the ethical standards as laid down in the 1964 Declaration of Helsinki and its later amendments.

Informed consent Informed consent was obtained from all individual participants included in the study.

Open Access This article is licensed under a Creative Commons Attribution-NonCommercial 4.0 International License, which permits any non-commercial use, sharing, adaptation, distribution and reproduction in any medium or format, as long as you give appropriate credit to the original author(s) and the source, provide a link to the Creative Commons licence, and indicate if changes were made. The images or other third party material in this article are included in the article's Creative Commons licence, unless indicated otherwise in a credit line to the material. If material is not included in the article's Creative Commons licence and your intended use is not permitted by statutory regulation or exceeds the permitted use, you will need to obtain permission directly from the copyright holder. To view a copy of this licence, visit http://creativecommons.org/licenses/by-nc/4.0/.

\section{References}

1. Lip GYH, Collet JP, Haude M, Byrne R, Chung EH, Fauchier L, et al. 2018 Joint European consensus document on the management of antithrombotic therapy in atrial fibrillation patients presenting with acute coronary syndrome and/or undergoing percutaneous cardiovascular interventions: a joint consensus document of the European Heart Rhythm Association (EHRA), European Society of Cardiology Working Group on Thrombosis, European Association of Percutaneous Cardiovascular Interventions (EAPCI), and European Association of Acute Cardiac Care (ACCA) endorsed by the Heart Rhythm Society (HRS), AsiaPacific Heart Rhythm Society (APHRS), Latin America Heart Rhythm Society (LAHRS), and Cardiac Arrhythmia Society of Southern Africa (CASSA). Europace. 2019;21(2):192-3.

2. Levine GN, Bates ER, Bittl JA, Brindis RG, Fihn SD, Fleisher LA, et al. 2016 ACC/AHA guideline focused update on duration of dual antiplatelet therapy in patients with coronary artery disease: a report of the American College of Cardiology/American Heart Association Task Force on clinical practice guidelines: an update of the 2011 ACCF/AHA/SCAI guideline for percutaneous coronary intervention, 2011 ACCF/AHA guideline for coronary artery bypass graft surgery, 2012 ACC/AHA/ACP/AATS/PCNA/ SCAI/STS guideline for the diagnosis and management of patients with stable ischemic heart disease, 2013 ACCF/AHA guideline for the management of ST-elevation myocardial infarction, 2014 AHA/ACC guideline for the management of patients with nonST-elevation acute coronary syndromes, and 2014 ACC/AHA guideline on perioperative cardiovascular evaluation and management of patients undergoing noncardiac surgery. Circulation. 2016;134(10):e123-55.

3. Lai KC, Lam SK, Chu KM, Wong BC, Hui WM, Hu WH, et al. Lansoprazole for the prevention of recurrences of ulcer complications from long-term low-dose aspirin use. N Engl J Med. 2002;346(26):2033-8. 
4. Cardoso RN, Benjo AM, DiNicolantonio JJ, Garcia DC, Macedo FY, El-Hayek G, et al. Incidence of cardiovascular events and gastrointestinal bleeding in patients receiving clopidogrel with and without proton pump inhibitors: an updated meta-analysis. Open Heart. 2015;2(1):e000248.

5. Hulot JS, Collet JP, Silvain J, Pena A, Bellemain-Appaix A, Barthelemy O, et al. Cardiovascular risk in clopidogrel-treated patients according to cytochrome P450 2C19*2 loss-of-function allele or proton pump inhibitor coadministration: a systematic meta-analysis. J Am Coll Cardiol. 2010;56(2):134-43.

6. Goodman SG, Clare R, Pieper KS, Nicolau JC, Storey RF, Cantor WJ, et al. Association of proton pump inhibitor use on cardiovascular outcomes with clopidogrel and ticagrelor: insights from the platelet inhibition and patient outcomes trial. Circulation. 2012;125(8):978-86.

7. Nicolau JC, Bhatt DL, Roe MT, Lokhnygina Y, Neely B, Corbalan $\mathrm{R}$, et al. Concomitant proton-pump inhibitor use, platelet activity, and clinical outcomes in patients with acute coronary syndromes treated with prasugrel versus clopidogrel and managed without revascularization: insights from the Targeted Platelet Inhibition to Clarify the Optimal Strategy to Medically Manage Acute Coronary Syndromes trial. Am Heart J. 2015;170(4):683-94.e3.

8. Sherwood MW, Melloni C, Jones WS, Washam JB, Hasselblad $\mathrm{V}$, Dolor RJ. Individual proton pump inhibitors and outcomes in patients with coronary artery disease on dual antiplatelet therapy: a systematic review. J Am Heart Assoc. 2015;4(11):e002245.

9. Shah NH, LePendu P, Bauer-Mehren A, Ghebremariam YT, Iyer $\mathrm{SV}$, Marcus J, et al. Proton pump inhibitor usage and the risk of myocardial infarction in the general population. PLoS One. 2015;10(6):e0124653.

10. Bhatt DL, Cryer BL, Contant CF, Cohen M, Lanas A, Schnitzer TJ, et al. Clopidogrel with or without omeprazole in coronary artery disease. N Engl J Med. 2010;363(20):1909-17.

11. Ray WA, Chung CP, Murray KT, Smalley WE, Daugherty JR, Dupont WD, et al. Association of proton pump inhibitors with reduced risk of warfarin-related serious upper gastrointestinal bleeding. Gastroenterology. 2016;151(6):1105-12.e10.

12. Ray WA, Chung CP, Murray KT, Smalley WE, Daugherty JR, Dupont WD, et al. Association of oral anticoagulants and proton pump inhibitor cotherapy with hospitalization for upper gastrointestinal tract bleeding. JAMA. 2018;320(21):2221-30.

13. Chan EW, Lau WC, Leung WK, Mok MT, He Y, Tong TS, et al. Prevention of dabigatran-related gastrointestinal bleeding with gastroprotective agents: a population-based study. Gastroenterology. 2015;149(3):586-95.e3.

14. Sharma R, Roy A, Ramos C, Rosenberg R, Garcia-Carrasquillo $\mathrm{R}$, Lebwohl B. Low adherence to national guidelines for proton-pump inhibitor prescription in patients receiving combination aspirin and anticoagulation. Therap Adv Gastroenterol. 2017;10(5):387-96.

15. Center for Drug Evaluation and Research. Clinical pharmacology review NDA 22-512, dabigatran. https://www.accessdata.fda.gov/ drugsatfda_docs/nda/2010/022512Orig1s000ClinPharmR_Corrr ected\%203.11.2011.pdf. Accessed 19 Mar 2019.

16. Cannon CP, Bhatt DL, Oldgren J, Lip GYH, Ellis SG, Kimura T, et al. Dual antithrombotic therapy with dabigatran after PCI in atrial fibrillation. N Engl J Med. 2017;377(16):1513-24.

17. Cannon CP, Gropper S, Bhatt DL, Ellis SG, Kimura T, Lip GY, et al. Design and rationale of the RE-DUAL PCI trial: a prospective, randomized, phase $3 \mathrm{~b}$ study comparing the safety and efficacy of dual antithrombotic therapy with dabigatran etexilate versus warfarin triple therapy in patients with nonvalvular atrial fibrillation who have undergone percutaneous coronary intervention with stenting. Clin Cardiol. 2016;39(10):555-64.

18. Golwala HB, Cannon CP, Steg PG, Doros G, Qamar A, Ellis SG, et al. Safety and efficacy of dual vs. triple antithrombotic therapy in patients with atrial fibrillation following percutaneous coronary intervention: a systematic review and meta-analysis of randomized clinical trials. Eur Heart J. 2018;39(19):1726-35a.

19. Lopes RD, Hong H, Harskamp RE, Bhatt DL, Mehran R, Cannon CP, et al. Safety and efficacy of antithrombotic strategies in patients with atrial fibrillation undergoing percutaneous coronary intervention: a network meta-analysis of randomized controlled trials. JAMA Cardiol. 2019;4(8):747-55.

20. Hu W, Tong J, Kuang X, Chen W, Liu Z. Influence of proton pump inhibitors on clinical outcomes in coronary heart disease patients receiving aspirin and clopidogrel: a meta-analysis. Medicine (Baltimore). 2018;97(3):e9638

21. Vaduganathan M, Cannon CP, Cryer BL, Liu Y, Hsieh WH, Doros $\mathrm{G}$, et al. Efficacy and safety of proton-pump inhibitors in highrisk cardiovascular subsets of the COGENT trial. Am J Med. 2016;129(9):1002-5.

22. Vaduganathan M, Bhatt DL, Cryer BL, Liu Y, Hsieh WH, Doros $\mathrm{G}$, et al. Proton-pump inhibitors reduce gastrointestinal events regardless of aspirin dose in patients requiring dual antiplatelet therapy. J Am Coll Cardiol. 2016;67(14):1661-71.

23. Moayyedi P, Eikelboom JW, Bosch J, Connolly SJ, Dyal L, Shestakovska O, et al. Safety of proton pump inhibitors based on a large, multi-year, randomized trial of patients receiving rivaroxaban or aspirin. Gastroenterology. 2019;157(3):682-91.e2.

24. Moayyedi P, Eikelboom JW, Bosch J, Connolly SJ, Dyal L, Shestakovska O, et al. Pantoprazole to prevent gastroduodenal events in patients receiving rivaroxaban and/or aspirin in a randomized, double-blind, placebo-controlled trial. Gastroenterology. 2019;157(2):403-12.e5.

25. Connolly SJ, Ezekowitz MD, Yusuf S, Eikelboom J, Oldgren J, Parekh A, et al. Dabigatran versus warfarin in patients with atrial fibrillation. N Engl J Med. 2009;361(12):1139-51.

26. Desai J, Kolb JM, Weitz JI, Aisenberg J. Gastrointestinal bleeding with the new oral anticoagulants: defining the issues and the management strategies. Thromb Haemost. 2013;110(2):205-12.

27. Steffel J, Verhamme P, Potpara TS, Albaladejo P, Antz M, Desteghe L, et al. The 2018 European Heart Rhythm Association practical guide on the use of non-vitamin $\mathrm{K}$ antagonist oral anticoagulants in patients with atrial fibrillation. Eur Heart J. 2018;39(16):1330-93.

28. Vaduganathan M, Bhatt DL. Gastrointestinal bleeding with oral anticoagulation: understanding the scope of the problem. Clin Gastroenterol Hepatol. 2017;15(5):691-3.

29. Eikelboom JW, Wallentin L, Connolly SJ, Ezekowitz M, Healey JS, Oldgren J, et al. Risk of bleeding with 2 doses of dabigatran compared with warfarin in older and younger patients with atrial fibrillation: an analysis of the randomized evaluation of long-term anticoagulant therapy (RE-LY) trial. Circulation. 2011;123(21):2363-72.

30. Cangemi DJ, Krill T, Weideman R, Cipher DJ, Spechler SJ, Feagins LA. A comparison of the rate of gastrointestinal bleeding in patients taking non-vitamin $\mathrm{K}$ antagonist oral anticoagulants or warfarin. Am J Gastroenterol. 2017;112(5):734-9.

31. Agewall S, Cattaneo M, Collet JP, Andreotti F, Lip GY, Verheugt $\mathrm{FW}$, et al. Expert position paper on the use of proton pump inhibitors in patients with cardiovascular disease and antithrombotic therapy. Eur Heart J. 2013;34(23):1708-13, 13a-13b.

32. Kuwayama T, Osanai H, Ajioka M, Tokuda K, Ohashi H, Tobe $\mathrm{A}$, et al. Influence of proton pump inhibitors on blood dabigatran concentrations in Japanese patients with non-valvular atrial fibrillation. J Arrhythm. 2017;33(6):619-23.

33. Oldgren J, Steg PG, Hohnloser SH, Lip GYH, Kimura T, Nordaby M, et al. Dabigatran dual therapy with ticagrelor or clopidogrel after percutaneous coronary intervention in atrial fibrillation patients with or without acute coronary syndrome: a 
subgroup analysis from the RE-DUAL PCI trial. Eur Heart J. 2019;40(19):1553-62.

34. Bundhun PK, Teeluck AR, Bhurtu A, Huang WQ. Is the concomitant use of clopidogrel and proton pump inhibitors still associated with increased adverse cardiovascular outcomes following coronary angioplasty? A systematic review and meta-analysis of recently published studies (2012-2016). BMC Cardiovasc Disord. 2017;17(1):3.
35. O’Donoghue ML, Braunwald E, Antman EM, Murphy SA, Bates ER, Rozenman Y, et al. Pharmacodynamic effect and clinical efficacy of clopidogrel and prasugrel with or without a protonpump inhibitor: an analysis of two randomised trials. Lancet. 2009;374(9694):989-97.

\section{Affiliations}

José C. Nicolau ${ }^{1}$ (1) - Deepak L. Bhatt ${ }^{2}$ - Stefan H. Hohnloser ${ }^{3} \cdot$ Takeshi Kimura $^{4}$. Gregory Y. H. Lip ${ }^{5,6}$. Corinna Miede ${ }^{7}$. Matias Nordaby ${ }^{8}$. Jonas Oldgren ${ }^{9}$. Philippe Gabriel Steg ${ }^{10,11}$. Jurriën M. ten Berg ${ }^{12}$ - Lucas C. Godoy ${ }^{1,13}$. Christopher P. Cannon ${ }^{2}$ on behalf of The RE-DUAL PCI Steering Committee and Investigators

1 Instituto do Coracao (InCor), Hospital das Clínicas HCFMUSP, Faculdade de Medicina, Universidade de Sao Paulo, Enéas Carvalho Aguiar, 44, Sao Paulo, SP 05403-000, Brazil

2 Brigham and Women's Hospital and Heart and Vascular Center, and Harvard Medical School, Boston, MA, USA

3 Johann Wolfgang Goethe University, Frankfurt am Main, Germany

4 Kyoto University, Kyoto, Japan

5 Liverpool Centre for Cardiovascular Science, University of Liverpool and Liverpool Heart and Chest Hospital, Liverpool, UK

6 Aalborg Thrombosis Research Unit, Department of Clinical Medicine, Aalborg University, Aalborg, Denmark

7 Mainanalytics ma GmbH, Sulzbach (Taunus), Germany
8 Boehringer Ingelheim International $\mathrm{GmbH}$, Ingelheim am Rhein, Germany

9 Uppsala Clinical Research Center, and Department of Medical Sciences, Uppsala University, Uppsala, Sweden

10 Université de Paris, FACT, INSERM U_1148, Paris, France

11 Hôpital Bichat, Assistance Publique-Hôpitaux de Paris, Paris, France

12 St. Antonius Ziekenhuis, Nieuwegein, the Netherlands

13 Peter Munk Cardiac Centre, University of Toronto, Toronto, ON, Canada 